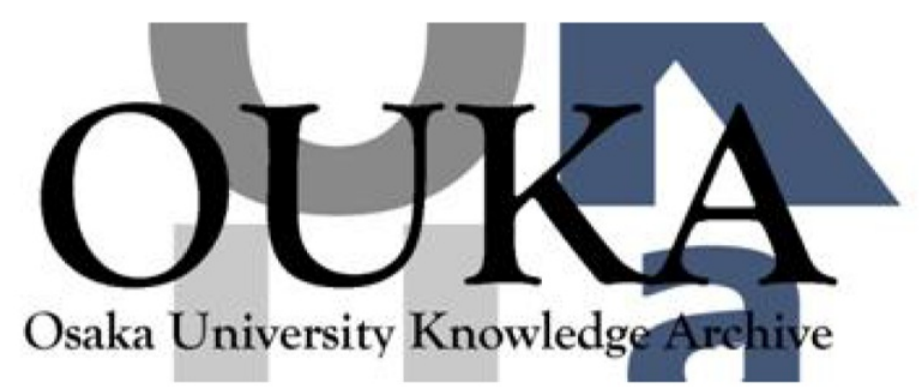

\begin{tabular}{|c|l|}
\hline Title & Wave Dispersion Relations in Yukawa Fluids \\
\hline Author(s) & Ohta, H.; Hamaguchi, S. \\
\hline Citation & Physical Review Letters. 84(26) p. 6026-p. 6029 \\
\hline Issue Date & $2000-06-26$ \\
\hline oaire:version & VoR \\
\hline URL & https://hdl. handle. net/11094/78495 \\
\hline rights & $\begin{array}{l}\text { Copyright (2000) by the American Physical } \\
\text { Society. }\end{array}$ \\
\hline Note & \\
\hline
\end{tabular}

Osaka University Knowledge Archive : OUKA

https://ir. Library. osaka-u. ac. jp/

Osaka University 


\title{
Wave Dispersion Relations in Yukawa Fluids
}

\author{
H. Ohta and S. Hamaguchi \\ Department of Fundamental Energy Science, Kyoto University, Gokasho, Uji, Kyoto 611-0011, Japan
}

(Received 18 October 1999; revised manuscript received 11 May 2000)

\begin{abstract}
The wave dispersion relations in the fluid phase of Yukawa systems are obtained from molecular dynamics (MD) simulations for a wide range of the parameters. The Yukawa system is a collection of particles interacting through Yukawa (i.e., screened Coulomb) potentials, which can serve as a simple model for dusty plasmas. Our simulations have clearly shown that the transverse wave dispersion has a cutoff at a long wavelength even in the case of weak screening. The MD simulation data are compared with earlier theoretical predictions of the Yukawa dispersion relations and the validity of these theories is clarified.
\end{abstract}

PACS numbers: 52.25.Ub, 52.25.Zb, 52.35.-g

The Yukawa system is a collection of particles interacting through Yukawa (i.e., screened Coulomb) pair potentials

$$
\phi(r)=\frac{Q^{2}}{4 \pi \varepsilon_{0}} \frac{\exp \left(-k_{D} r\right)}{r} .
$$

Here $r$ is the distance between two Yukawa particles and $k_{D}^{-1}$ is the screening length. As a mathematical model, the Yukawa system may be of special interest as its behavior can be varied continuously between systems governed by short-range and long-range forces. As to specific physical systems, Yukawa systems may serve as a model for charged dust particles in plasmas or colloidal particles in electrolytes [1-6]. In a plasma, the charge on a dust particle (whose diameter is typically $1 \mu \mathrm{m}$ or less) is usually negative (which we denote by $-Q$ : typically a few thousand electron charges) as the mobility of electrons is much higher than that of ions. The screening is due to the formation of a Debye sheath around each charged dust particle and $k_{D}^{-1}$ is given by the Debye length of the background plasma. Recent experiments $[7,8]$ have demonstrated that such charged dust particles in plasmas support longitudinal wave excitations, which are called the dust acoustic waves (DAWs) [9-15].

When the Yukawa system is in thermodynamical equilibrium, it may be characterized by two dimensionless parameters: $\kappa=k_{D} a$, i.e., the ratio of the interparticle distance $a=(3 / 4 \pi n)^{1 / 3}$ (where $n$ is the particle number density) to the screening length $k_{D}^{-1}$ and $\Gamma=Q^{2} / 4 \pi \varepsilon_{0} a T$, i.e., the inverse of the system temperature (thermal energy) $T$ measured in units of $Q^{2} / 4 \pi \varepsilon_{0} a$. The system is called "strongly coupled" if the coupling parameter $\Gamma^{*}=\Gamma \exp (-\kappa)$-i.e., the ratio of the average interparticle potential energy to the average kinetic energy - is greater than unity. In the limit of no screening (i.e., $\kappa=0$ ), the system is called the one-component plasma (OCP) [16-20].

In this paper, we study dispersion relations of both longitudinal and transverse waves in the fluid phase of Yukawa systems, using molecular dynamics (MD) simulations [2-6]. The longitudinal wave of a Yukawa fluid may be regarded as the simplest approximation of the DAW, upon which one can build more realistic models of experimentally observed DAWs with, e.g., dust-neutral collisions. Recently several theoretical descriptions of the Yukawa dispersion relations have been presented $[10-13,15]$ in the strongly coupled regime. We shall compare our simulation results with some of these theories.

Let us consider a system of $N$ identical, infinitesimally small dust particles of mass $m$ and charge $-Q$. The force on each dust particle can be derived from the potential (i.e., "excess") energy $U_{\text {ex }}$, which is actually the total Helmholtz free energy of the background plasma for given instantaneous positions of dust particles [5]. After dropping the constant free energy of the uniform background plasma for simplicity, the excess energy may be written as $U_{\text {ex }}=U_{c}-N Q^{2} k_{D} / 8 \pi \varepsilon_{0}$ where

$$
U_{c}=\frac{1}{2} \sum_{i \neq j} \sum_{j} \phi\left(\left|\mathbf{r}_{i}-\mathbf{r}_{j}\right|\right)-\frac{N Q^{2} n}{2 \varepsilon_{0} k_{D}^{2}}
$$

is called the correlation energy. In Eq. (1), $\mathbf{r}_{i}$ and $\mathbf{r}_{j}$ denote the positions of $i$ th and $j$ th dust particles and the second term on the right-hand side represents the free energy (excluding the uniform ideal-gas free energy) of the chargeneutralizing background plasma. The remaining term in $U_{\text {ex }}$, i.e., $-N Q^{2} k_{D} / 8 \pi \varepsilon_{0}$, represents the free energy of each sheath. For details of the derivation of $U_{\mathrm{ex}}$, the reader is referred to Ref. [5].

Simulation particles are placed in a cubic box with periodic boundary conditions. The total potential is then calculated for the Yukawa interactions among all the particles in the simulation box and all of their periodic images. The infinite potential sum due to all periodic images (i.e., the Ewald sum) is approximated by a tensor-product spline function [21], which enables us to simulate Yukawa systems of small $\kappa$ efficiently with a relatively small number of the simulation particles $N[3,4]$. Thermodynamical equilibrium at a given temperature (or $\Gamma$ ) is first attained by periodically renormalizing particle velocities [4]. Once the system is in thermodynamical equilibrium, we discontinue 
the periodic velocity renormalization and let the system evolve under the constant-energy conditions. The statistical average \langle\rangle of a dynamical quantity is obtained as the time average over a sufficiently long time period in this microcanonical simulation.

Waves excited in Yukawa systems are collective motions of the constituent particles and can be characterized by space-time correlation functions. The longitudinal and transverse current correlation functions $[20,22]$ are defined as

$$
C_{l}(\mathbf{k}, t)=\frac{1}{N}\langle[\mathbf{k} \cdot \mathbf{j}(\mathbf{k}, t)][\mathbf{k} \cdot \mathbf{j}(-\mathbf{k}, 0)]\rangle
$$

and

$$
C_{t}(\mathbf{k}, t)=\frac{1}{2 N}\langle[\mathbf{k} \times \mathbf{j}(\mathbf{k}, t)] \cdot[\mathbf{k} \times \mathbf{j}(-\mathbf{k}, 0)]\rangle,
$$

where $\mathbf{j}(\mathbf{k}, t)=\sum_{j=1}^{N} \mathbf{v}_{i}(t) \exp \left[i \mathbf{k} \cdot \mathbf{r}_{j}(t)\right]$ is the Fourier transformation of the particle current for a given wave number vector $\mathbf{k}$. We also write the Fourier transformation of these functions into the frequency space as $\tilde{C}_{l}(\mathbf{k}, \omega)$ and $\tilde{C}_{t}(\mathbf{k}, \omega)$.

The combinations of $(\mathbf{k}, \omega)$ that give the peaks of $\tilde{C}_{l}(\mathbf{k}, \omega)$ and $\tilde{C}_{t}(\mathbf{k}, \omega)$ determine the dispersion relations for the longitudinal and transverse modes. Figures 1, 2, and 3 show the dispersion relations for $\kappa=0.1,1.0$, and 2.0 , respectively, obtained in this manner from MD simulations with $N=250$ and 800 in the fluid phase near solidification [2]. The abscissa represents the normalized wave number $q=k a$ with $k=|\mathbf{k}|$. The frequency is normalized by the nominal plasma frequency of Yukawa particles $\omega_{p}=\sqrt{Q^{2} n / \varepsilon_{0} m}$. The frequency resolution in all the figures in this paper is $\Delta \omega / \omega_{p}=0.0283$. For each $\kappa$, the

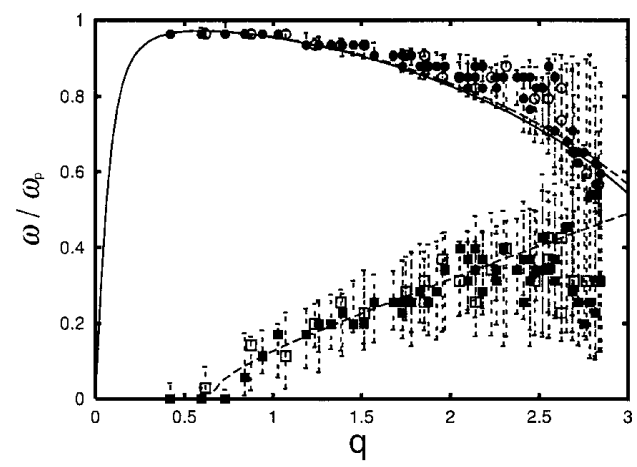

FIG. 1. The dispersion relations of the longitudinal $(\boldsymbol{O}, \bigcirc)$ and transverse $(\square, \square)$ waves obtained from MD simulations for $\kappa=0.1$. MD simulations with $N=800(\mathbf{0}, \mathbf{\square})$ and $N=$ $250(\bigcirc, \square)$ are for $\Gamma=150$ and $\Gamma=142$. The vertical bars represent the width of the intervals where the current correlation functions are larger than $75 \%$ of the maximum values. The solid curve is the dispersion based on the QLCA theory, representing Eq. (4) with $\kappa=0.1$ and $\Gamma=150$. The upper dashed curve represents Eq. (5) with $\Gamma=150$. The lower dashed curve is the least-squares fit of Eq. (6) to the transverse MD data for $0.5<q<2.0$ with the fitting parameters of $\left(\tau_{R} \omega_{p}\right)^{-1}=0.22$ and $\eta^{*}=0.13$. system temperatures (or $\Gamma$ ) are slightly different between the two sets of runs with $N=800$ and $N=250$ since we do not have precise control over temperatures in microcanonical simulations. In these runs, the temperatures fluctuate in time and the indicated temperatures are the time average in thermodynamical equilibrium. The dispersions obtained from these two sets of runs show good agreement, which implies the dispersion relations are hardly affected by the slight temperature differences and 250-particle MD simulations provide sufficiently accurate results.

Rosenberg and Kalman [11] obtained an analytic expression of the wave dispersion relation for the longitudinal mode in the strong-coupling regime, using the quasilocalized charge approximation (QLCA) [23,24]. In the longwavelength limit, it may be written as

$$
\begin{aligned}
\frac{\omega^{2}}{\omega_{p}^{2}}= & \frac{q^{2}}{q^{2}+\kappa^{2}} \\
& +\frac{q^{2}}{\Gamma}\left[\frac{4}{45} u_{c}-\frac{2}{45} y \frac{\partial u_{c}}{\partial y}+\frac{4}{15} y^{2} \frac{\partial^{2} u_{c}}{\partial y^{2}}\right],
\end{aligned}
$$

where $u_{c}=U_{c} / T$ is the normalized correlation energy and $y=\kappa^{2}$.

The solid curves in Figs. 1, 2, and 3 show the dispersion relations of Eq. (4) with $\kappa$ and $\Gamma$ being those for the corresponding $N=800$ simulations. For $\kappa \leq 1, u_{c}(\Gamma, \kappa)$ given in Refs. [3] and [4] was used to evaluate Eq. (4), as in Ref. [11]. For $\kappa=2$, the data given in Table VIII of Ref. [2] were used to evaluate $u_{c}(\Gamma, \kappa)$ and its derivatives. These curves are in good agreement with the MD simulation results.

The QLCA method to derive Eq. (4) is based on the premise that the wave motion is sufficiently fast that the average position of each oscillating Yukawa particle does not change significantly during the wave motion and each particle is subject to the average restoring force from other particles. In the fluid phase, however, if the wave motion is sufficiently slow, average positions of all particles can relax to new positions during the wave motion and the

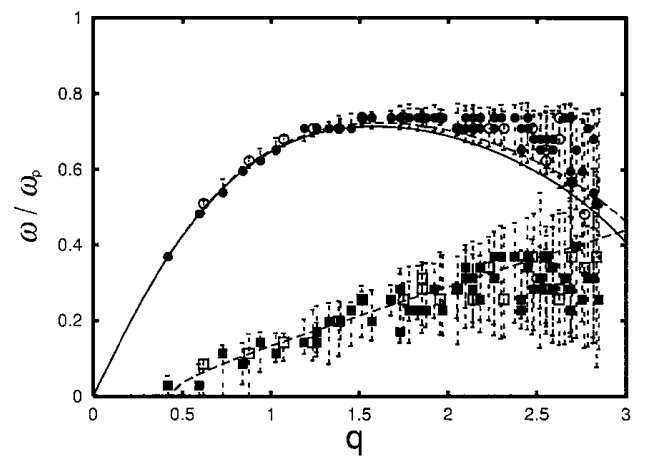

FIG. 2. The dispersion relations of the longitudinal and transverse waves obtained from MD simulations for $\kappa=1.0$ with $N=800$ (at $\Gamma=207$ ) and $N=250(\Gamma=202)$. The symbols are the same as those used in Fig. 1. The lower dashed curve is the least-squares fit of Eq. (6) to the transverse MD data for $0.5<q<2.0$ with the fitting parameters of $\left(\tau_{R} \omega_{p}\right)^{-1}=0.13$ and $\eta^{*}=0.16$. 


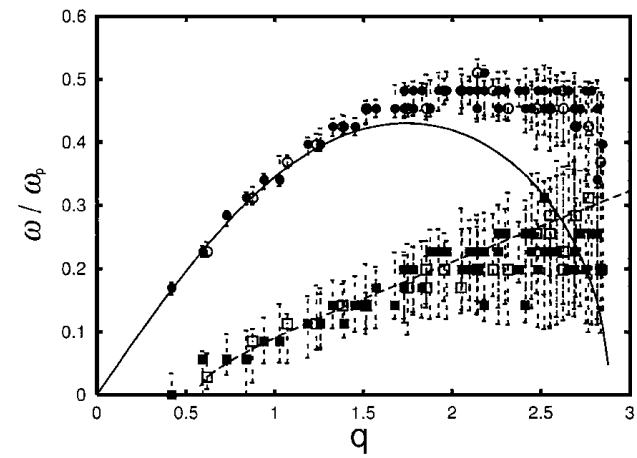

FIG. 3. The dispersion relations of the longitudinal and transverse waves obtained from MD simulations for $\kappa=2.0$ with $N=800$ (at $\Gamma=435)$ and $N=250(\Gamma=395)$. The symbols are the same as those used in Fig. 1. The lower dashed curve is the least-squares fit of Eq. (6) to the transverse MD data for $0.5<q<2.0$ with the fitting parameters of $\left(\tau_{R} \omega_{p}\right)^{-1}=0.12$ and $\eta^{*}=0.097$.

restoring force may not be fully exerted on each particle. This relaxation time of particle positions is called the "diffusion time" $\tau_{D}$ in Refs. [11] and [24]. The QLCA theory is thus applicable only to waves satisfying $\omega \tau_{D}>1$.

Recently Kaw and Sen have derived the wave dispersion relations of Yukawa systems using the generalizedhydrodynamic $(\mathrm{GH})$ equation [13]. The $\mathrm{GH}$ equation is the hydrodynamic equation incorporating the nonlocal viscoelasticity with memory effects arising from the strong correlation among constituent particles. The time scale of the phenomenological viscoelastic memory effect is specified by the "relaxation time" $\tau_{R}$ (denoted as $\tau_{m}$ in Ref. [13]), which we assume to be of the same order as the diffusion time $\tau_{D}$ of the QLCA theory. In the long-wavelength limit, the longitudinal-mode dispersion relation obtained from the GH equations [13] may be written for $\omega \tau_{R} \gg 1$ (i.e., kinetic regime) as

$$
\frac{\omega^{2}}{\omega_{p}^{2}}=\frac{q^{2}}{q^{2}+\kappa^{2}}+\frac{q^{2}}{\Gamma}\left(\frac{1}{3}+\frac{4}{45} u_{\mathrm{ex}}\right),
$$

where $u_{\mathrm{ex}}=U_{\mathrm{ex}} / T$ denotes the normalized excess energy. [This equation is the same as Eq. (19) of Ref. [13].] Since the GH equations used in Ref. [13] were originally constructed for OCPs, the dependence of $u_{\mathrm{ex}}$ on $\kappa$ was ignored in the derivation of Eq. (5). Also, in contrast with Eq. (4), there is a constant term $\left(\frac{1}{3}\right)$ in the parentheses of Eq. (5). The upper dashed curves in Figs. 1 and 2 depict the dispersion relation of Eq. (5) with the OCP excess energy $u_{\mathrm{ex}}$ from Ref. [18] (as suggested in Ref. [13]) with $\Gamma$ being those of the corresponding $N=800$ simulations. These dashed curves are almost identical with corresponding solid curves, indicating the differences between Eqs. (4) and (5) are negligible for $\kappa \leqslant 1$. In Fig. 3 (for $\kappa=2$ ), the nominal evaluation of Eq. (5) with the OCP excess energy $u_{\mathrm{ex}}$ is not presented as the resulting dispersion relation is completely off the MD simulation data. This indicates that, in the GH model, the correct evaluation of thermodynamical variables for Yukawa systems with finite $\kappa$ (rather than the OCP system) is essential.
The dispersion relation for the transverse mode (which is also called the shear mode) was also derived by Kaw and Sen from the GH equations and given in Eq. (21) of Ref. [13]. Writing the real and imaginary parts separately, we have

$$
\left(\frac{\operatorname{Re} \omega}{\omega_{p}}\right)^{2}=\frac{\eta^{*}}{\tau_{R} \omega_{p}} q^{2}-\frac{1}{4\left(\tau_{R} \omega_{p}\right)^{2}}
$$

and $\operatorname{Im} \omega=1 / 2 \tau_{R}$ for the mode satisfying $q^{2}>$ $1 / 4 \tau_{R} \omega_{p} \eta^{*}$ and arbitrary $\omega \tau_{R}$. Here $\eta^{*}=\left(\frac{4}{3} \eta+\zeta\right) /$ $\left(m n \omega_{p} a^{2}\right)$ is the normalized kinematic longitudinal viscosity with $\eta$ and $\zeta$ being the shear and bulk viscosities [22].

Ideally one should estimate $\tau_{R}$ and $\eta^{*}$ directly from MD simulations. However, this would be a major computational task that requires detailed analysis of the assumed phenomenological viscoelastic memory function. Instead here we take a simple alternative approach: We now ask, if Eq. (6) is valid, what numerical values should the thermodynamical parameters $\tau_{R}$ and $\eta^{*}$ take? To answer this question, we least-squares fit Eq. (6) to the transversemode dispersion data (denoted by $\mathbf{\square}$ and $\square$ ) in Figs. 1-3. As our simulation results clearly show that there is a cutoff for the transverse dispersion around $q \simeq 0.5$ (even in the weak screening case of $\kappa=0.1$ ), we need to exclude such data points from fitting. In addition, hydrodynamics theory is valid only for long-wavelength modes, so we have used data only on $0.5<q<2$ for fitting. (Simulation data of $N=800$ and $N=250$ agree well despite the small differences in temperature, so we have used both data sets for a single fitting.) The lower dashed curves in Figs. 1-3 represent these fitting curves, where the fitting parameters are $\left(\tau_{R} \omega_{p}\right)^{-1}=0.22,0.13,0.12$, and $\eta^{*}=0.13,0.16,0.097$, respectively. These $\tau_{R}$ values imply that the longitudinalwave dispersion relation of Eq. (4) indeed satisfies the kinetic regime condition $\omega \tau_{D} \gg 1$ (under the assumption of $\tau_{R} \simeq \tau_{D}$ ), which justifies the use of the QLCA method in the derivation of Eq. (4).

If the particle correlations are weak, then $\tau_{R}$ is small and the system is in the hydrodynamic regime $\left(\omega \tau_{R} \ll 1\right)$. In this case, the dispersion relation of the longitudinal mode may be derived from the standard Navier-Stokes equations as

$$
\left(\frac{\operatorname{Re} \omega}{\omega_{p}}\right)^{2}=\frac{q^{2}}{q^{2}+\kappa^{2}}+\frac{c_{s}^{2} q^{2}}{\omega_{p}^{2} a^{2}}-\frac{\eta^{* 2} q^{4}}{4}
$$

and $\operatorname{Im} \omega=-\eta^{*} k^{2} / 2$ with the sound velocity $c_{s}$. For inviscid fluids (i.e., $\eta^{*}=0$ ), Eq. (7) represents the Langmuir wave dispersion for the OCP (i.e., $\kappa=0$ ) and the standard sound-wave dispersion in the case of highly screened neutral gases (i.e., $\kappa \rightarrow \infty$ ). In the isothermal ideal gas case, one may set $c_{s}=c_{0} \equiv(3 T / m)^{1 / 2}$. Note that $c_{0}^{2} / \omega_{p}^{2} a^{2}=1 / \Gamma$. The shear mode cannot be sustained in the weakly coupled regime.

Figures 4 and 5 show the dispersion relations of the longitudinal mode in Yukawa fluids when the coupling is moderate: $\Gamma=1.04, \kappa=0.1\left(\Gamma^{*}=0.94\right)$ for Fig. 4 , and $\Gamma=1.98, \kappa=1.0\left(\Gamma^{*}=0.73\right)$ for Fig. 5, obtained from 


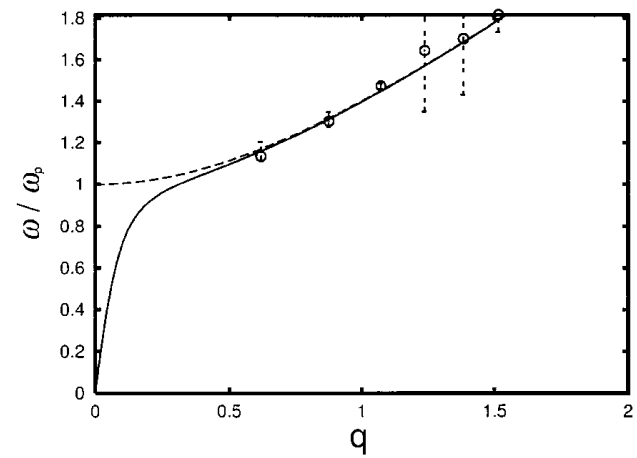

FIG. 4. As in the previous figures, the open circles $\bigcirc$ with bars represent the dispersion relation of the longitudinal mode at $\kappa=$ $0.1, \Gamma=1.04\left[\Gamma^{*} \equiv \Gamma \exp (-\kappa)=0.94\right]$ obtained from MD simulations with $N=250$. The solid curve represents Eq. (7) with $\eta^{*}=0$ and $c_{s}=c_{0}$. The dashed curve is the dispersion relation of the Langmuir wave, i.e., Eq. (7) with $\kappa=\eta^{*}=0$ and $c_{s}=c_{0}$.

MD simulations with $N=250$. In Fig. 4 the solid curve represents Eq. (7) with $c_{s}=c_{0}$ and $\eta^{*}=0$, which shows excellent agreement with the MD data. The Langmuir wave dispersion relation [i.e., Eq. (7) with $\kappa=\eta^{*}=0$ and $c_{s}=c_{0}$ ] is also given by the dashed curve for reference. Since Eq. (7) with $c_{s}$ estimated from the OCP excess energy (as suggested in Ref. [13]) disagrees with our MD simulation data for larger $\kappa$, we again least-squares fit Eq. (7) to the simulation data in Fig. 5. The obtained fitting parameters are $c_{s}^{2} / c_{0}^{2}=0.63$ and $\eta^{*}=0$, which indicates that this system has a higher compressibility than that with a smaller $\kappa$.

In summary, we have performed MD simulations and obtained the wave dispersion relations of Yukawa fluids in the parameter range that covers both strong and weak coupling. It is of particular interest that there is a cutoff of the transverse wave dispersion at a long wavelength ( $q \simeq 0.5$ for $\kappa \lesssim 2$ near solidification). Such cutoffs are known for neutral fluids governed by short-range forces, but in previous studies for plasmalike systems with longerrange forces, including Ref. [20] for OCPs, the presence of transverse wave cutoff had never been shown. This also

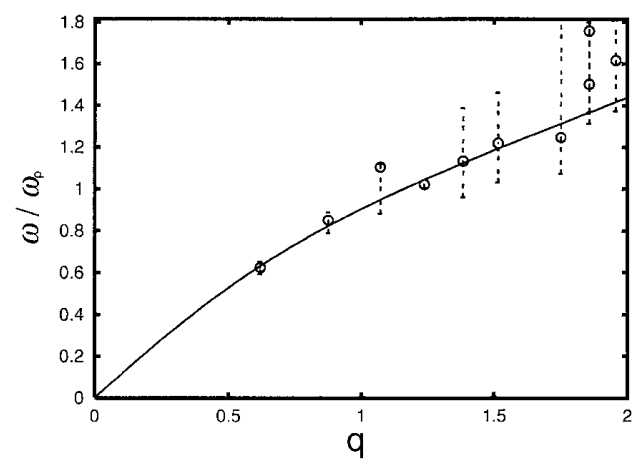

FIG. 5. The dispersion relation of the longitudinal mode at $\kappa=1.0, \Gamma=1.98\left(\Gamma^{*}=0.73\right)$. The symbols are the same as those in the previous figure. The solid curve represents the leastsquares fit of Eq. (7) to the MD simulation data, which provides the fitting parameters $\eta^{*}=0$ and $c_{s}^{2}=0.63 c_{0}$. suggests that, in order to identify the dust shear (i.e., transverse) wave experimentally, one has to seek modes with relatively short wavelengths and low frequencies. We have also compared our numerically obtained Yukawa dispersion relations with some of the earlier theoretical estimates $[11,13]$ and found good agreement in the regime where their stated approximations are valid. We expect the dispersion relations derived from the GH model in Ref. [13] can be further improved for larger $\kappa$ if the thermodynamical variables (such as the excess energy $u_{\mathrm{ex}}$ ) are correctly evaluated for the corresponding Yukawa system with finite $\kappa$.

The authors thank R.T. Farouki, who developed the original version of the MD code, for helpful discussions on numerical simulations. This work was in part supported by the Sumitomo Foundation.

[1] U. Konopka, G. E. Morfill, and L. Ratke, Phys. Rev. Lett. 84, 891 (2000).

[2] S. Hamaguchi, R. T. Farouki, and D. H. E. Dubin, Phys. Rev. E 56, 4671 (1997).

[3] S. Hamaguchi, R. T. Farouki, and D. H. E. Dubin, J. Chem. Phys. 105, 7641 (1996).

[4] R. T. Farouki and S. Hamaguchi, J. Chem. Phys. 101, 9885 (1994).

[5] S. Hamaguchi and R. T. Farouki, J. Chem. Phys. 101, 9876 (1994).

[6] M. O. Robbins, K. Kremer, and G. S. Grest, J. Chem. Phys. 88, 3286 (1988).

[7] A. Barkan, R. L. Merlino, and N. D’Angelo, Phys. Plasmas 2, 3565 (1995).

[8] J. B. Pieper and J. Goree, Phys. Rev. Lett. 77, 3137 (1996).

[9] N. N. Rao, P. K. Shukla, and M. Y. Yu, Planet. Space Sci. 38, 543 (1990).

[10] X. Wang and A. Bhattacharjee, Phys. Plasmas 4, 3759 (1997).

[11] M. Rosenberg and G. Kalman, Phys. Rev. E 56, 7166 (1997).

[12] M. S. Murillo, Phys. Plasmas 5, 3116 (1998).

[13] P. K. Kaw and A. Sen, Phys. Plasmas 5, 3552 (1998).

[14] D. Winske, M. S. Murillo, and M. Rosenberg, Phys. Rev. E 59, 2263 (1999).

[15] M. S. Murillo, Phys. Plasmas 7, 33 (2000).

[16] S. G. Brush, H. L. Sahlin, and E. Teller, J. Chem. Phys. 45, 2102 (1966).

[17] M. Baus and J.-P. Hansen, Phys. Rep. 59, 1 (1980).

[18] W. L. Slattery, G. D. Doolen, and H.E. DeWitt, Phys. Rev. A 21, 2087 (1980); 26, 2255 (1982).

[19] R. T. Farouki and S. Hamaguchi, Phys. Rev. E 47, 4330 (1993).

[20] P. Schmidt, G. Zwicknagel, P.-G. Reinhard, and C. Toepffer, Phys. Rev. E 56, 7310 (1997).

[21] R. T. Farouki and S. Hamaguchi, J. Comput. Phys. 115, 276 (1994).

[22] J. P. Hansen and I. R. McDonald, Theory of Simple Liquids (Academic Press, London, 1986).

[23] G. Kalman and K. I. Golden, Phys. Rev. A 41, 5516 (1990).

[24] K. I. Golden, G. Kalman, and P. Wyns, Phys. Rev. A 46, 3454 (1992). 